\title{
Evolution and Fate of the Residual Body of Toxoplasma gondii revealed by FIB- SEM series
}

\author{
Marcia Attias ${ }^{1,2}$, Kildare Miranda ${ }^{1,2}$ and Wanderley de Souza ${ }^{1,2}$
}

${ }^{1}$ Instituto de Biofisica Carlos Chagas Filho, Universidade Federal do Rio de Janeiro, Rio de Janeiro, Brazil

${ }^{2}$ Instituto Nacional de Ciência e Tecnologia em Biologia Estrutural e Bioimagens, UFRJ, RJ. Brazil

Toxoplasma gondii, the causative agent of toxoplasmosis, is an intracellular protozoan parasite belonging to the phylum Apicomplexa. T. gondii can invade and multiply in virtually any nucleated cell of birds and mammals, including man. Once invasion is complete, T. gondii starts to divide by endodyogeny inside a membrane limited parasitophorous vacuole (PV) in the cytoplasm of the host cell. The endodyogenic cycles proceed and as the daughter cells remain attached to the residual body (RB), the whole assembly resembles a rosette, with parasites pointing their apical ends towards the vacuolar membrane. At a certain point, parasites detach from the rosette and egress from the vacuole and the host cell, destroying it [1]. Although it plays a key role in maintaining the rosette assembly of tachyzoites, little attention has been given to the residual body and some authors consider it as a compartment where the leftover of the parasites is stored.

In order to investigate the development and fate of the RB and what role could be associated to it besides to organize the daughter cells in a rosette shape, we used both transmission and scanning electron microscopy. However, a timeline of events starting with the appearance and reaching the final fate of the RB could be traced from careful observation of several series of FIB-SEM slice and view from LLCMK2 Cells infected with the RH strain of T. gondii for up to 48hours. All samples were chemically fixed with $2.5 \%$ glutaraldehyde in $0.1 \mathrm{M} \mathrm{pH} 7.2$ cacodylate buffer, post fixed in $1 \% \mathrm{OsO}_{4}$ in the same buffer plus $1.25 \%$ potassium ferrocyanide and either critical point dried and sputtered with gold or embedded in Polybed resin and polymerized for $48 \mathrm{~h}$ at $60^{\circ} \mathrm{C}$. The SEM samples were dry scraped with Scotch $($ tape before sputtering and observed in a JEOL6340 field emission SEM. The TEM ultrathin sections were observed in a ZEISS900 TEM and The slice and view FIB series were obtained either on a FEI-Helios nanolab or in a ZEISS Auriga dual beam SEM. 3D models were generated with the IMOD package [2].

Our hypothesis is that the RB is not a storage compartment of leftover organelles that were not inserted in daughter cells. It is certain that it helps to maintain the assembly of tachyzoites as a rosette, optimizing the occupation of the PV and the position of the apical complex for egress. However, as only 3-D reconstruction of FIB-SEM slice and view series could show, the mitochondrial profiles seen inside it are actually part of the mother cell mitochondria that was not yet separated and inserted in the daughter cells (Figs. A, E and F). In some dividing cells, a structure formerly identified as a nucleus, was shown to be a spherical electrondense structure involved by a double membrane profile, which is consistent with the description of an autophagosome, probably containing the mother cell rhoptries. In 24 to 48hpi the RB of mature rosettes contained almost exclusively acidocalcisome like vacuoles (Figs. A, B, C, D, H). These showed clear signals of fusion Fig. D, G, H, I), and at the final stage, when the host cell was already lysed, they could be seen fusing with the RB membrane, no more with parasites attached to it (Fig. H). 
Taken together, these observations lead us to propose that the RB is a site of progressive storage of acidocalcisomes that will ultimately fuse, causing detachment of parasites adhered to it, liberation of large quantities of calcium and phosphates in the PV space and its self destruction.

\section{References:}

[1] Blader I.J. et al. Annual review of microbiology, vol. 69, 2015 p. 463.

[2] Kremer, J.R. et al Journal of structural biology, vol. 116, no. 1, p. 71 .

The authors acknowledge funding from the Conselho Nacional de Desenvolvimento Científico e Tecnológico, Fundação Carlos Chagas de Amparo à Pesquisa.

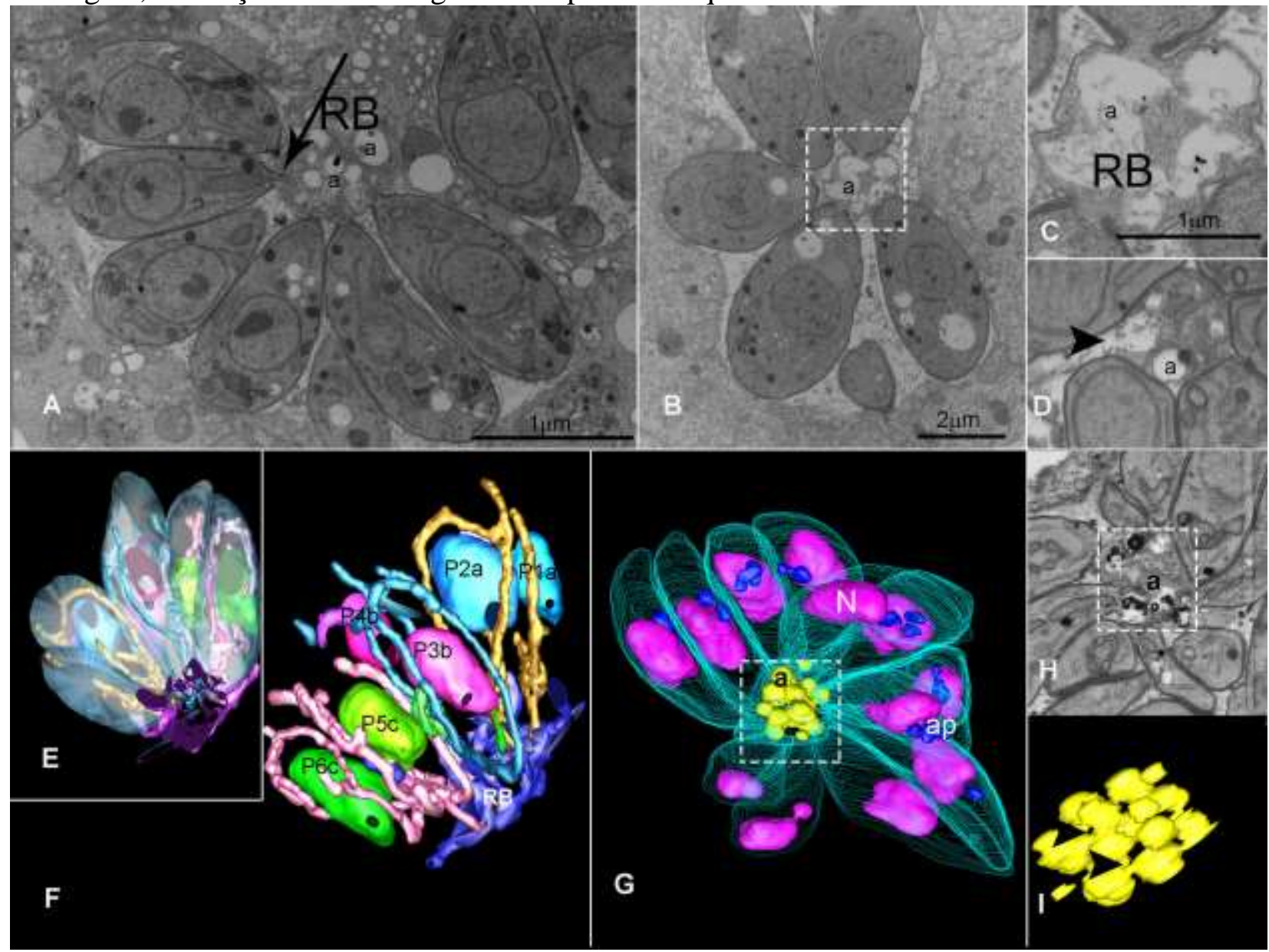

Figures: A, B and C TEM ultrathin sections of a rosette of tachyzoites attached to the RB. Inside it, acidocalcisomes (a). A 24hpi: a mitochondrial branch is seen in the RB entering the parasite (arrow). B 48hpi: the acidocalcisomes fusing to each other. C zoom view of the area limited by the dotted square in B. D slice of a FIB-SEM series showing an acidocalcisome opening in the PV space (arrowhead). E and F models showing the connections between the mitochondria of each two parasites (P1 to P6), nuclei and mitochondria of sister cells have the same color or letter ( $\mathrm{a}, \mathrm{b}$ or $\mathrm{C})$. G Model from a series. The RB is filled with acidocalcisomes (a) in process of fusion. $\mathbf{H}$ representative slice from the series used to build the model. Parasites nuclei (N) in magenta and apicoplasts (ap) in blue I zoom view of the area inside the dotted square in G. Arrowheads point to the fusion sites. 